\title{
Limiting Performance of Millimeter-Wave Communications in the Presence of a 3D Random Waypoint Mobility Model
}

\author{
Massimiliano Comisso, Francesca Vatta, Giulia Buttazzoni, and Fulvio Babich
}

\begin{abstract}
This paper proposes a mathematical framework for evaluating the limiting capacity of a millimeter-wave (mmWave) communication involving a mobile user (MU) and a cellular base station. The investigation is realized considering a threedimensional (3D) space in which the random waypoint mobility model is used to probabilistically identify the location of the MUs. Besides, the analysis is developed accounting for path-loss attenuation, directional antenna gains, shadowing, and modulation scheme. Closed-form formulas for the received signal power, the Shannon capacity, and the bit error rate (BER) are obtained for both line-of-sight (LoS) and non-LoS scenarios in the presence of a noise-limited operating regime. The conceived theoretical model is firstly checked by Monte Carlo validations, and then employed to explore the influence of the antenna gain and of the cell radius on the capacity and on the BER of a fifth-generation (5G) link in a 3D environment, taking into account both the 28 and $73 \mathrm{GHz}$ mmWave bands.
\end{abstract}

Index Terms-5G communications; millimeter-waves; Shannon capacity; random waypoint mobility model.

\section{INTRODUCTION}

For telecommunication scientists and engineers, 2020 is awaited to be the year in which the definite accomplishment of the fifth-generation $(5 \mathrm{G})$ cellular system worldwide will take place, with a blending of real and virtual innovative components. To obtain this accomplishment, an ultra-densification of the base stations (BSs) will be required, in conjunction with the design of transceivers operating at tenths of gigahertz. Moreover, another requirement will be the virtualization of the core and edge network functionalities. These requirements are needed to build an ultra-high capacity and very low-latency pervasive network capable to also incorporate the current fourth-generation (4G) Long-Term Evolution (LTE) system and the existing gigabit Wireless-Fidelity (WiFi) technology [1]-[16], with the aim of finally realizing the for a while conceived Internet of Things (IoT) and Internet of Everything (IoE) paradigms.

Manuscript received December 30, 2020; revised March 5, 2021. Date of publication March 19, 2021. Date of current version March 19, 2021.

Authors are with the DIA, University of Trieste, Trieste, Italy (e-mails mcomisso@units.it, vatta@units.it, gbuttazzoni@units.it, babich@units.it).

Part of this work was presented at the International Conference on Software,

Telecommunications and Computer Networks, SoftCOM '20, Hvar, Croatia,

September 17-19, 2020.

This work is partly supported by the Italian Ministry of University and Research within project FRA 2018 (Univ. of Trieste, Italy) "UBER-5G: Cubesat 5G networks - Access layer analysis and antenna system development."

Digital Object Identifier (DOI): 10.24138/jcomss-2020-0008
The concrete implementation of the 5G technology, together with its exploratory evaluation and realization, is at a starting point, whereas its conceptual analysis, as far as its attainable performance is concerned, is instead a mature research field, with several relevant studies that have been published in last years [17]-[25]. Namely, the $60 \mathrm{GHz}$ links have been analyzed in [17] developing a mathematical set-up considering the modeling of the antenna pattern through a linear array of flat-top elements. Moreover, [18] presents a coverage analysis to derive a simpler model of dense networks. Furtherly, [19] addresses multi-tier millimeter-wave (mmWave) networks, considering pragmatic propagation environments, pursuing the evaluation of their coverage probability. Alternatively, [20] investigates the starting access, addressing in deep detail the search and interference management problems. A general channel power distribution, considering disparate statistics, is used in [21] to analyze the performance limits of BS densification in multidimensional mmWave networks. [22] considers the effect of the interferers spatial distribution on the capture probability, whereas, in [23], the use of arrays with few elements is proved to rise the strength against the beam alignment errors employing the analysis of the coverage. [24] analyzes the influence of the spatial statistic on network performance adopting a precise propagation model and [25] theoretically estimates the statistic of the bit error rate (BER) extending the results of [24]. Finally, the problem of exploiting the vacant beams available at a BS is addressed in [26], where the author proposes a method to employ them for doubling the throughput of the adjacent active MU-BS links.

A frequent characteristic of the above mentioned works is the spatial statistic assumed to express the position of the mobile users (MUs) and/or of the BSs. Actually, these studies contemplate the commonly employed spatial Poisson point process (PPP), having the significant merit of conducting to analytically tractable models when the space domain is not bounded. A small number of studies, parallel to the PPP, consider uncommon spatial statistics, such as the uniform distribution (UD) and the random waypoint mobility model (RWMM). As far as these two distributions are concerned, the UD has obtained more consideration [27], [28], whereas the RWMM has been unfrequently adopted for $5 \mathrm{G}$ applications [22], [24]. This latter model is of a certain interest because of its extensive employment in network simulators where it is used to delineate the random motion of a nodes set in a 
bounded space from the statistical point of view [29]. Being an alternative choice with respect to the PPP, its adoption for $5 \mathrm{G}$ applications may constitute a fascinating topic because of the original understanding of the $5 \mathrm{G}$ context it can provide. Moreover, it is certainly more realistic in the $5 \mathrm{G}$ environment because the actual BS position is not subject to a pre-defined statistic, but is related to the actual shape and height of the surrounding buildings and foliage. This attribute becomes more important when taking into account the more realistic three-dimensional (3D) scenario instead of the usual twodimensional (2D) one to study the $5 \mathrm{G}$ performance. This more realistic scenario is certainly more appropriate to model the classic 5G small-cell context, where two communicating nodes are supposed to be positioned one over the other. This may occur when a BS and a MU are located at different floors of a building, or when two remotely-controlled flying vehicles, so called unmanned aerial vehicles (UAVs), fly in a 3D formation. Thus, the investigation of the capacity of a $5 \mathrm{G}$ mmWave communication link in the presence of a 3D RWMM may produce relevant outcomes, complementing those obtained applying the ordinary PPP-based mathematical frameworks.

In [30] we have addressed this topic presenting a theoretical evaluation of a $5 \mathrm{G}$ communication link performance in the presence of a 3D RWMM. The study is performed in a noiselimited regime by assuming state-of-the-art 3D channel models [31], including path-loss attenuation, directional antennas, and mid-scale fading for both line-of-sight (LoS) and non-LoS (NLoS) link-state conditions.

The original contribution of the present paper is related to the consideration of the $3 \mathrm{D}$ scenario and to the assumption of the RWMM, and represents an extension of our previous works [22], considering exclusively the 2D environment and [24], focusing on the PPP to evaluate the Shannon capacity. In particular, exact analytical expressions for the probability density function (pdf) and the cumulative distribution function (cdf) of the received power and of the channel capacity are derived and validated through Monte Carlo simulations. The obtained results are used to study the influence of the transmitting/receiving antenna gains and of the cell radius on the behavior of a $5 \mathrm{G}$ communication for the 28 and $73 \mathrm{GHz}$ mmWave bands. As compared to [30], the here proposed theoretical framework presents analytical formulas for the average signal-to-noise ratio (SNR) and, subsequently, for the corresponding BER in agreement with the adopted modulation scheme. The results obtained by this extension enable to infer the influence of the product gain on the error performance when the MUs are located according to a 3D RWMM.

The paper is organized as follows. Section II introduces the addressed scenario. Section III describes the bit error rate (BER) analysis, while Section IV illustrates the derived error performance. Finally, Section V summarizes the most relevant conclusions.

Notation. Throughout the paper the following notation is used: $\mathbb{R}_{>0}$ and $\mathbb{R}_{\geq 0}$ denote the sets of positive and nonnegative reals, respectively; $\mathbb{1}_{\mathbf{X}}(\mathbf{x})$ denotes the indicator function (i.e., $\mathbb{1}_{\mathbf{X}}(\mathbf{x})=1$ if $\mathbf{x} \in \mathbf{X}, \mathbb{1}_{\mathbf{X}}(\mathbf{x})=0$ if $\mathbf{x} \notin \mathbf{X}$ ); $\operatorname{erfc}(x)$ denotes the complementary error function; $Q(x)$ denotes the
$Q$-function.

\section{Node Location ANd CHANNEL Models}

Assume a 5G cellular network where a mmWave communication link is established between a BS and a MU located in a finite $3 \mathrm{D}$ region around the $\mathrm{BS}$. More exactly, the $\mathrm{BS}$ is positioned at the center $O$ of a $3 \mathrm{D}$ ball $\mathbf{B}(O, \rho)$ of radius $\rho$, using the RWMM to model the MU site.

In [32] was obtained the pdf of the random variable (r.v.) $R$, defined as the distance of a point from the center of the ball, for the 3D scenario. From this result, the pdf of the BS-MU distance can be expressed as:

$$
f_{R}(r)=\left[\sum_{k=1}^{3} a_{k}(2 k+1) \frac{r^{2 k}}{\rho^{2 k+1}}\right] \mathbb{1}_{\mathbb{R}_{\geq 0}}(r),
$$

where $a_{1}=245 / 72, a_{2}=-119 / 36$, and $a_{3}=65 / 72$ are the coefficients of the statistic [32]. The resulting cdf of $R$ can be calculated by integrating (1) on $r$, thus obtaining:

$$
F_{R}(r)= \begin{cases}0 & r<0 \\ \sum_{k=1}^{3} a_{k}\left(\frac{r}{\rho}\right)^{2 k+1} & 0 \leq r \leq \rho \\ 1 & r>\rho\end{cases}
$$

The RWMM is implemented in ns 2 and GloMoSim simulation tools for evaluating the performance of wireless networks and communication protocols. Assuming this spatial statistic, the MU selects a position located inside the ball and then proceeds in its direction at a certain speed. The selection of both the location and the speed is performed randomly on the basis of a UD. Once the MU reaches the selected position, it stands by for a while and afterwards selects randomly another position and another moving speed. In steady-state conditions, that is, when the number of movements approaches infinity, the cdf of $R$ asymptotically approaches (2), which represents a nonuniform distribution independent of the moving speed [29].

The latest experimental exploration of the extremely high frequency (EHF) band has disclosed the most important factors characterizing the mmWave channel. These are the noise power and the link-state [31], determining the path-loss attenuation and the mid-scale fading statistic [17]. As far as the link-state is concerned, the communication is feasible in two conditions, namely the LoS and NLoS ones. The further link-state condition, called outage, models the absence of any link due to an infinite path-loss. For LoS/NLoS states, the omnidirectional path-loss attenuation is linearly dependent from the logarithm of the distance $R$, and thus can be expressed as:

$$
L(R)=\frac{1}{\alpha R^{\beta}}
$$

being $\alpha$ the the floating intercept and $\beta$ the average path-loss exponent. The link-state and the operating mmWave frequency band determine both these latter constants. For the 28 and $73 \mathrm{GHz}$ bands, their precise determination has been performed in [31], through a best-fit linear regression of the acquired experimental data. 
The link-state and the operating mmWave frequency band affects also the mid-scale fading statistic, which, in contrast with what happened in the $2-4 \mathrm{G}$ cellular systems, is much more relevant than small-scale fading for the $5 \mathrm{G}$ mmWave system. In particular, shadowing may be modeled by a r.v. $\Xi$ following a log-normal distribution, whose pdf can be written as [22]:

$$
f_{\Xi}(\xi)=\frac{1}{\sqrt{2 \pi} \tilde{\sigma} \xi} \exp \left(-\frac{\log ^{2} \xi}{2 \tilde{\sigma}^{2}}\right) \mathbb{1}_{\mathbb{R}_{>0}}(\xi),
$$

where $\tilde{\sigma}$ is the shadowing standard deviation.

Together with the link-state, the path-loss attenuation, and the mid-scale fading, the additional important propagation phenomenon influencing a mmWave communication performance is the noise power, which can be expressed as [19]:

$$
\mathcal{N}=\mathcal{N}_{0} \cdot W \cdot \mathcal{F}
$$

where $\mathcal{N}_{0} \cong 3.98 \cdot 10^{-21} \mathrm{~W} / \mathrm{Hz}$ is the noise spectral density, while $W$ and $\mathcal{F}$ are, respectively, the bandwidth and the noise figure of the receiver. The significance of the noise power points out an additional dissimilarity between the $\mu$ Wave 2$4 \mathrm{G}$ cellular networks and the mmWave $5 \mathrm{G}$ one. The first ones, as a matter of fact, are normally interference-limited, whereas the latter one is frequently noise-limited. The last hypothesis is valid if the communication is directional enough and the node densities are low enough [17].

\section{BER ANALYSIS}

The expressions derived during the development of the capacity analysis in [30] may be suitably exploited to estimate the BER of a mmWave communication link in agreement with the selected modulation scheme. To this aim, one can firstly evaluate the average SNR from the previously obtained statistics to subsequently calculate the corresponding BER. Accordingly, the average SNR $\mathbf{E}[\Omega]$ may be determined by using (16) in [30] and then exploiting the linearity of the expectation operator, thus obtaining:

$$
\begin{aligned}
\mathbf{E}[\Omega] & =\mathbf{E}\left[\frac{P}{\mathcal{N}}\right]=\frac{\mathbf{E}[P]}{\mathcal{N}} \\
& =\frac{\mathbf{E}[T \Xi]}{\mathcal{N}}=\frac{\mathbf{E}[T] \mathbf{E}[\Xi]}{\mathcal{N}},
\end{aligned}
$$

in which the average received power $\mathbf{E}[P]$ is expressed, by remembering (11) in [30], as the product between the average values of the independent r.v.s $T$ and $\Xi$. In particular, $\mathbf{E}[T]$ may be calculated as:

$$
\begin{aligned}
\mathbf{E}[T] & =\int_{-\infty}^{+\infty} t f_{T}(t) \mathrm{d} t=\int_{-\infty}^{+\infty} t \frac{\mathrm{d} F_{T}}{\mathrm{~d} t} \mathrm{~d} t \\
& =\int_{-\infty}^{+\infty} t\left[\frac{1}{t \beta} \sum_{k=1}^{3}(2 k+1) a_{k}\left(\frac{\delta}{t}\right)^{\frac{2 k+1}{\beta}}\right] \mathbb{1}_{[\delta,+\infty]}(t) \mathrm{d} t \\
& =\frac{1}{\beta} \sum_{k=1}^{3}(2 k+1) a_{k} \int_{\delta}^{+\infty}\left(\frac{\delta}{t}\right)^{\frac{2 k+1}{\beta}} \mathrm{d} t
\end{aligned}
$$

$$
=\delta \sum_{k=1}^{3} \frac{(2 k+1) a_{k}}{2 k+1-\beta}
$$

by using (9) in [30]. This latter evaluation maintains its significance until $\beta<3$, an assumption that, according to the experimental channel characterization presented in [31], is verified in both $\mathrm{LoS}$ and NLoS mmWave propagation scenarios. Regarding the second expectation required by (6), the average value of the r.v. $\Xi$ may be obtained from:

$$
\begin{aligned}
\mathbf{E}[\Xi] & =\int_{-\infty}^{+\infty} \xi f_{\Xi}(\xi) \mathrm{d} \xi \\
& =\frac{1}{\sqrt{2 \pi} \tilde{\sigma}} \int_{0}^{+\infty} \exp \left(-\frac{\log ^{2} \xi}{2 \tilde{\sigma}^{2}}\right) \mathrm{d} \xi \\
& =\exp \left(\frac{\tilde{\sigma}^{2}}{2}\right)
\end{aligned}
$$

by remembering (4). Since a noise-limited regime has been assumed throughout the proposed analysis, the availability of the average SNR allows the investigation of the corresponding BER according to the selected modulation scheme. To this purpose, with reference to a modulation of order $M$, one may focus the attention on two of the more widely adopted schemes: the $M$-phase-shift keying (PSK) modulation and the $M$ - quadrature amplitude modulation (QAM). By following the approximations provided in [33] and [34], a joint analytical formulation may be adopted to describe the BER of these two schemes. In particular, this formulation allows one to estimate the BER as:

$$
\mathcal{E} \cong \varsigma_{M} Q\left(\chi_{M} \sqrt{\mathbf{E}[\Omega]}\right)
$$

where the parameters $\varsigma_{M}$ and $\chi_{M}$, which account for the specific constellation, are defined as:

$$
\begin{gathered}
\varsigma_{M}=\frac{2}{\log _{2} M} \cdot \begin{cases}1 & M-\mathrm{PSK} \\
2\left(1-\frac{1}{\sqrt{M}}\right) & M-\mathrm{QAM}\end{cases} \\
\chi_{M}=\sqrt{\log _{2} M} \cdot \begin{cases}\sqrt{2} \sin \left(\frac{\pi}{M}\right) & M-\mathrm{PSK} \\
\sqrt{\frac{3}{M-1}} & M-\mathrm{QAM}\end{cases}
\end{gathered}
$$

By replacing (6) and then (7) and (8) in (9), one finally obtains the BER estimation as:

$$
\mathcal{E} \cong \varsigma_{M} Q\left[\chi_{M} \sqrt{\frac{\delta}{\mathcal{N}} \exp \left(\frac{\tilde{\sigma}^{2}}{2}\right) \sum_{k=1}^{3} \frac{(2 k+1) a_{k}}{2 k+1-\beta}}\right]
$$

which, similarly to the other provided expressions, is derived in analytical form. The numerical values obtainable from (11) are illustrated and discussed in the next section. 
TABLE I

DEFAULTS PARAMETER VALUES ADOPTED FOR TRANSMISSION POWER, ANTENNA GAINS, CELL RADIUS, BANDWIDTH, NOISE FIGURE, AND CHANNEL PARAMETERS (FLOATING INTERCEPT, PATH-LOSS EXPONENT, SHADOWING STANDARD DEVIATION) IN LOS/NLOS CONDITIONS FOR THE TWO MMWAVE BANDS [31].

\begin{tabular}{|l|l||l|l||l|l|}
\hline$P_{\mathrm{T}}$ & $100 \mathrm{~mW}$ & $G_{\mathrm{T}}$ & $10 \mathrm{dBi}$ & $G_{\mathrm{R}}$ & $10 \mathrm{dBi}$ \\
\hline$\rho$ & $100 \mathrm{~m}$ & $W$ & $1 \mathrm{GHz}$ & $\mathcal{F}$ & 10 \\
\hline & $61.4 \mathrm{~dB}(28 \mathrm{GHz}, \mathrm{LOS})$ & & $2(28 \mathrm{GHz}, \mathrm{LOS})$ & & $5.8 \mathrm{~dB}(28 \mathrm{GHz}, \mathrm{LOS})$ \\
$\alpha$ & $72.0 \mathrm{~dB}(28 \mathrm{GHz}, \mathrm{NLOS})$ & & $2.92(28 \mathrm{GHz}, \mathrm{NLOS})$ & $\tilde{\sigma}$ & $8.7 \mathrm{~dB}(28 \mathrm{GHz}, \mathrm{NLOS})$ \\
& $69.8 \mathrm{~dB}(73 \mathrm{GHz}, \mathrm{LOS})$ & $\beta$ & $2(73 \mathrm{GHz}, \mathrm{LOS})$ & & $5.8 \mathrm{~dB}(73 \mathrm{GHz}, \mathrm{LOS})$ \\
& $82.7 \mathrm{~dB}(73 \mathrm{GHz}, \mathrm{NLOS})$ & & $2.69(73 \mathrm{GHz}, \mathrm{NLOS})$ & & $6.7 \mathrm{~dB}(73 \mathrm{GHz}, \mathrm{NLOS})$ \\
\hline
\end{tabular}

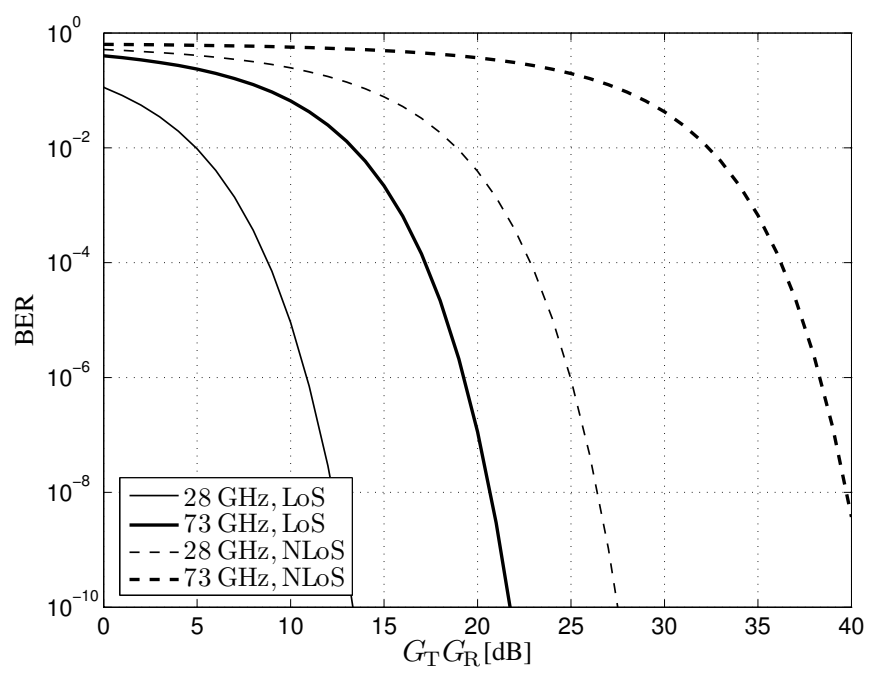

(a) $\mathcal{E}$ for $16-\mathrm{QAM}$

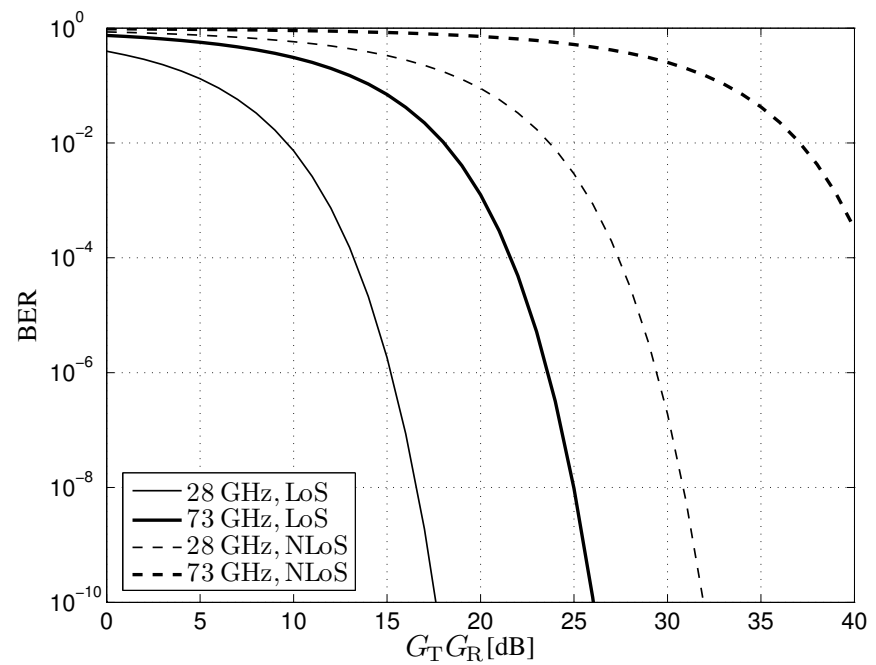

(c) $\mathcal{E}$ for $16-\mathrm{PSK}$

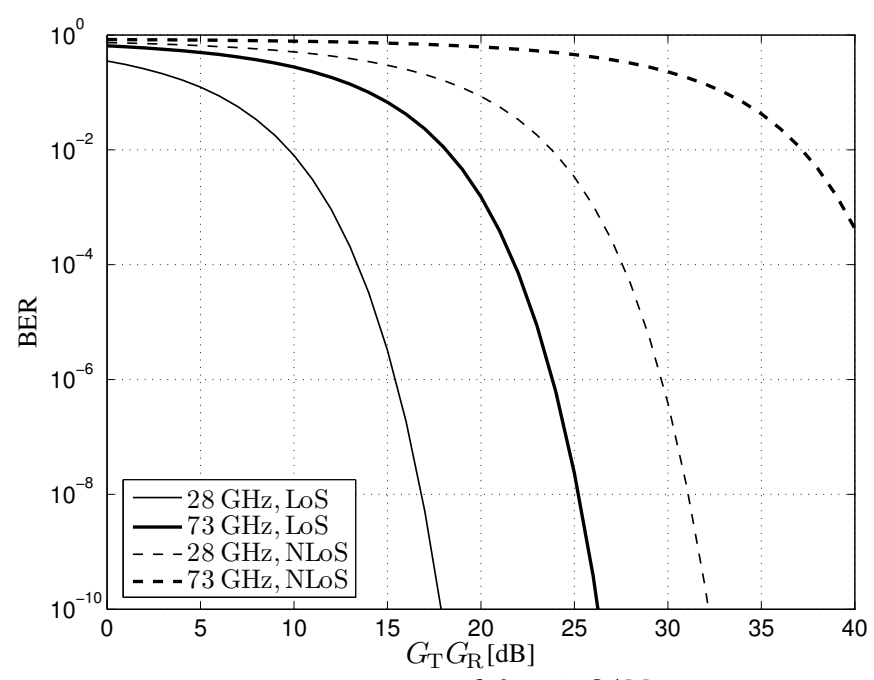

(b) $\mathcal{E}$ for $64-\mathrm{QAM}$

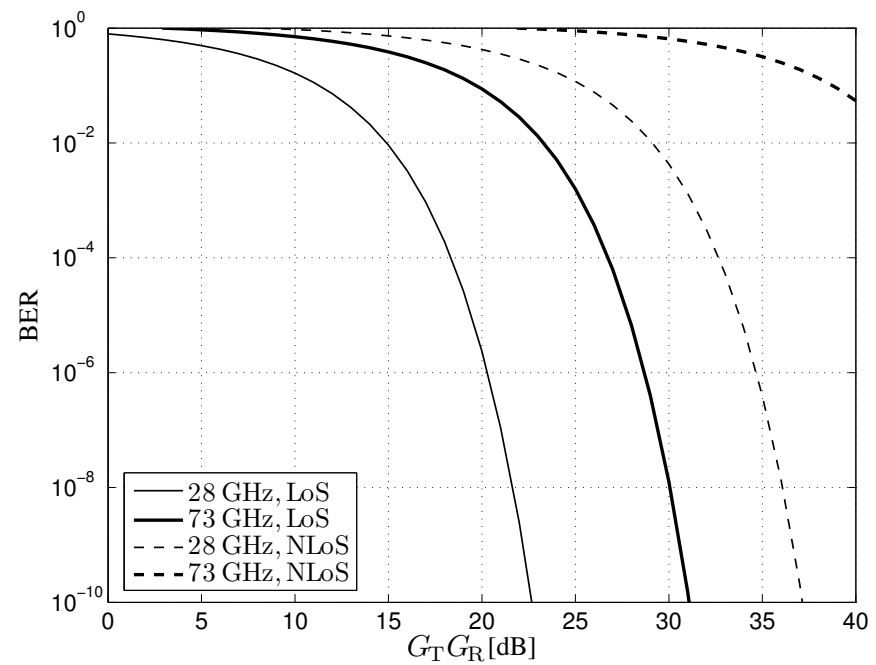

(d) $\mathcal{E}$ for $32-\mathrm{PSK}$

Fig. 1. BER for the 28 and $73 \mathrm{GHz}$ channels in $\operatorname{LoS}$ and NLoS conditions as a function of the product antenna gain for $\rho=100$ m: (a) 16-QAM, (b) 64-QAM, (c) 16-PSK, (d) 32-PSK.

\section{BER RESULTS}

The presented BER results are evaluated using the parameters listed in Table I (where $P_{\mathrm{T}}$ is the transmission power, $G_{\mathrm{T}}$ is the transmitting antenna power gain of the $\mathrm{MU}$, and $G_{\mathrm{R}}$ is the receiving antenna power gain of the BS), which have been experimentally derived in [31] by extended measurement campaigns carried out in the 28 and $73 \mathrm{GHz}$ mmWave bands.
According to this table, if not otherwise stated, the default values for the cell radius $\rho$ and for the product antenna gain $G_{\mathrm{T}} G_{\mathrm{R}}$ will be set equal to $100 \mathrm{~m}$ and $20 \mathrm{~dB}$, respectively. Besides, all the presented results are obtained employing the Matlab tool and adopting a nonuniform discretization of the support of the analyzed r.v.s, in order to reduce the computational time necessary for evaluating their statistics.. More precisely, the derived curves are reported in Fig. 1, 
which shows the error performance as a function of the product antenna gain when the 16-QAM (Fig. 1(a)), 64QAM (Fig. 1(b)), 16-PSK (Fig. 1(c)), and 32-PSK (Fig. 1(d)) modulations are employed.

These figures confirm, for any band, link state, and modulation, that the BER decreases with the increase of the product antenna gain. This behavior may be explained by observing that higher $G_{\mathrm{T}} G_{\mathrm{R}}$ values directly determine higher SNRs. Furthermore, beside the expected higher BER in LoS conditions with respect to that in NLoS ones for a given modulation and mmWave band, one may specifically notice the very low performance that characterizes the $73 \mathrm{GHz}$ channel in the NLoS case. This aspect may be clarified in mathematical terms by jointly observing (7) and (10) in [30], from which one may infer that the increase of the average path-loss exponent $\beta$ has a relevant impact on the average power $\mathbf{E}[T]$ received at the BS in the absence of mobility. Similar arguments may be exploited to justify the better error performance achievable in the $28 \mathrm{GHz}$ LoS channel. With reference to this specific case, one may observe that the 16-QAM scheme becomes preferable when the target of the communication is identified by the integrity of the data, while the 64-QAM one is more appropriate when, instead, the transmission rate is the main concern. This is a direct consequence of the well known higher spectral efficiency of the $M$-QAM schemes as compared to that of the $M$-PSK ones under the assumption of identical modulation order.

\section{CONCLUSIONS}

In our preliminary work [30] we have evaluated the cdf and pdf of the received power and of the Shannon capacity for a $5 \mathrm{G}$ communication link by means of a mathematical framework. Assuming mmWave channel models, lately estimated through experimental measurement campaigns, in [30] we have derived closed-form analytical formulas for capacity evaluation, verified through numerical simulations. Here, also a BER analysis has been added together with the derived error performance. The influence of the operating EHF band and of the link state on the attainable performance has been investigated by means of the proposed analysis. In [30] and in the present paper, the antenna gains have been shown to be crucial for the robustness of the communication link both in LoS and NLoS conditions, although the most significant advantages can be obtained making use of the $28 \mathrm{GHz}$ band with not too large cell radii. The ongoing research pursues the extension of the obtained physical layer model to come up with a valid reception criterion for establishing the result of a packet transmission in an ultra-dense contention-based scenario. Another research direction that would be interesting to promote has to do with the presence of non-Gaussian noise and/or co-channel interference. Both these phenomena need a generalization of the SNR modeling approach based on suitable colored channel models, specially conceived for the 28 and $73 \mathrm{GHz}$ operating bands, in which the noise-limited conjecture is not valid any more.

\section{REFERENCES}

[1] J.G. Andrews, S. Buzzi, W. Choi, S.V. Hanly, A. Lozano, A.C.K. Soong, and J.C. Zhang, "What will 5G be?" IEEE Journal on Selected Areas in Communications, vol. 32, no. 6, pp. 1065-1082, June 2014.

[2] F. Babich and M. Comisso, "Multi-packet communication in heterogeneous wireless networks adopting spatial reuse: Capture analysis," IEEE Transactions on Wireless Communications, vol. 12, no. 10, pp. 53465359, Oct. 2013.

[3] K.C-J. Lin, S. Gollakota, and D. Katabi, "Random access heterogeneous MIMO networks," in ACM SIGCOMM Conference, Aug. 2011.

[4] P. Schulz, M. Matthé, H. Klessig, M. Simsek, G. Fettweis, J. Ansari, S.A. Ashraf, B. Almeroth, J. Voigt, I. Riedel, A. Puschmann, A. MitscheleThiel, M. Müller, T. Elste, and M. Windisch, "Latency critical IoT applications in 5G: Perspective on the design of radio interface and network architecture,' IEEE Communications Magazine, vol. 55, no. 2, pp. 70-78, Feb. 2017.

[5] F. Babich, M. Comisso, and A. Dorni, "A novel SIR-based access scheme for multi-packet communication in 802.11 networks," in IEEE International Conference on Communications (ICC), 10-15 June 2012, pp. $1-5$.

[6] K. Cengiz and M. Aydemir, "Next-generation infrastructure and technology issues in 5G systems," Journal of Communications Software and Systems, vol. 14, no. 1, pp. 33-39, Mar. 2018.

[7] F. Vatta, A. Soranzo, and F. Babich, "More accurate analysis of sumproduct decoding of LDPC codes using a Gaussian approximation," IEEE Communications Letters, vol. 23, no. 2, pp. 230-233, Feb. 2019.

[8] G.A. Akpakwu, B.J. Silva, G.P. Hancke, and A.M. Abu-Mahfouz, "A survey on $5 \mathrm{G}$ networks for the Internet of Things: Communication technologies and challenges," IEEE Access, vol. 6, pp. 3619-3647, Feb. 2018.

[9] S. Selmi and R. Bouallègue, "Spectral and energy efficient D2D communication underlay 5G networks: A mixed strategy approach," Journal of Communications Software and Systems, vol. 16, no. 1, pp. 57-65, Mar. 2020.

[10] F. Babich, M. Noschese, and F. Vatta, "Analysis and design of rate compatible LDPC codes," in IEEE International Symposium on Personal, Indoor and Mobile Radio Communications (PIMRC), 4-8 Sept. 2016, pp. 1-6.

[11] W. Hong, Z.H. Jiang, C. Yu, J. Zhou, P. Chen, Z. Yu, H. Zhang, B. Yang, X. Pang, M. Jiang, Y. Cheng, M.K.T. Al-Nuaimi, Y. Zhang, J. Chen, and S. He, "Multibeam antenna technologies for $5 \mathrm{G}$ wireless communications," IEEE Transactions on Antennas and Propagation, vol. 65, no. 12, pp. 6231-6249, Dec. 2017.

[12] A. Seetharaman, N. Niranjan, V. Tandon, S. Devarajan, M.K. Moorthy, and A.S. Saravanan, "What do customers crave in mobile 5G? A survey spotlights four standout factors," IEEE Consumer Electronics Magazine, vol. 6, no. 3, pp. 52-66, July 2017.

[13] R. Corrado, M. Comisso, and F. Babich, "On the impact of the video quality assessment in $802.11 \mathrm{e}$ ad-hoc networks using adaptive retransmissions," in IEEE IFIP Annual Mediterranean Ad Hoc Networking Workshop (Med-Hoc-Net), 2-4 June 2014, pp. 47-54.

[14] N.O. Parchin, M. Alibakhshikenari, H.J. Basherlou, R.A. AbdAlhameed, J. Rodriguez, and E. Limiti, "MM-wave phased array quasiYagi antenna for the upcoming 5G cellular communications," Applied Sciences, vol. 9, no. 5, pp. 1-14, Mar. 2019, Article ID9050978.

[15] A. Arvidsson and L. Westberg, "Transport bottlenecks of edge computing in 5G networks," Journal of Communications Software and Systems, vol. 15, no. 1, pp. 59-65, Mar. 2019.

[16] M. Comisso, G. Buttazzoni, and R. Vescovo, "Reconfigurable antenna arrays with multiple requirements: A versatile 3D approach," International Journal of Antennas and Propagation, vol. 2017, pp. 1-9, Article ID6752108.

[17] S. Singh, R. Mudumbai, and U. Madhow, "Interference analysis for highly directional $60-\mathrm{GHz}$ mesh networks: The case for rethinking medium access control," IEEE/ACM Transactions on Networking, vol. 19, no. 5, pp. 1513-1527, Oct. 2011.

[18] T. Bai and R.W. Heath Jr., "Coverage and rate analysis for millimeterwave cellular networks," IEEE Transactions on Wireless Communications, vol. 14, no. 2, pp. 1100-1114, Feb. 2015.

[19] M. Di Renzo, "Stochastic geometry modeling and analysis of multitier millimeter wave cellular networks," IEEE Transactions on Wireless Communications, vol. 14, no. 9, pp. 5038-5057, Sep. 2015.

[20] H. Shokri-Ghadikolaei, C. Fischione, G. Fodor, P. Popovski, and M. Zorzi, "Millimeter wave cellular networks: A MAC layer perspective," IEEE Transactions on Communications, vol. 63, no. 10, pp. 3437-3458, Oct. 2015. 
[21] V.M. Nguyen and M. Kountouris, "Performance limits of network densification," IEEE Journal on Selected Areas in Communications, vol. 35, no. 6, pp. 1294-1308, June 2017.

[22] F. Babich, M. Comisso, and A. Cuttin, "Impact of interference spatial distribution on line-of-sight millimeter-wave communications," in $V D E$ European Wireless (EW), 16-19 May 2017, pp. 363-368.

[23] M. Cheng, J.-B. Wang, Y. Wu, X.-G. Xia, K.-K. Wong, and M. Lin, "Coverage analysis for millimeter wave cellular networks with imperfect beam alignment," IEEE Transactions on Vehicular Technology, vol. 67, no. 9, pp. 8302-8314, Sep. 2018.

[24] M. Comisso and F. Babich, "Coverage analysis for 2D/3D millimeter wave peer-to-peer networks," IEEE Transactions on Wireless Communications, vol. 18, no. 7, pp. 3613-3627, July 2019.

[25] M. Comisso, F. Vatta, G. Buttazzoni, and F. Babich, "Estimation of the bit error rate (BER) for uplink millimeter-wave line-of-sight communications," in International Conference on Software, Telecommunications and Computer Networks (SoftCOM), 19-21 Sept. 2019, pp. 1-5.

[26] A.O.M. Al Janaby, "5G downlink throughput enhancement by beam consolidation at vacant traffic," Journal of Communications Software and Systems, vol. 15, no. 4, pp. 311-316, Dec. 2019.

[27] H. Inaltekin, M. Chiang, H.V. Poor, and S.B. Wicker, "On unbounded path-loss models: Effects of singularity on wireless network performance," IEEE Journal on Selected Areas in Communications, vol. 27, no. 7, pp. 1078-1092, Sep. 2009.

[28] V. Naghshin, A.M. Rabiei, N.C. Beaulieu, and B. Maham, "Accurate statistical analysis of a single interference in random networks with uniformly distributed nodes," IEEE Communications Letters, vol. 18, no. 2, pp. 197-200, Feb. 2014

[29] C. Bettstetter, G. Resta, and P. Santi, "The node distribution of the random waypoint mobility model for wireless ad hoc networks," IEEE Transactions on Mobile Computing, vol. 2, no. 3, pp. 257-269, July-Sep. 2003.

[30] M. Comisso, F. Vatta, G. Buttazzoni, and F. Babich, "Shannon capacity evaluation for $5 \mathrm{G}$ communications using the $3 \mathrm{D}$ random waypoint mobility model," in International Conference on Software, Telecommunications and Computer Networks (SoftCOM), 17-19 Sept. 2020, pp. $1-6$.

[31] M.R. Akdeniz, Y. Liu, M.K. Samimi, S. Sun, S. Rangan, T.S. Rappaport, and E. Erkip, "Millimeter wave channel modeling and cellular capacity evaluation," IEEE Journal on Selected Areas in Communications, vol. 32, no. 6, pp. 1164-1179, June 2014.

[32] E. Hyytiä and J. Virtamo, "Random waypoint model in n-dimensional space," Networking Laboratory, Helsinki University of Technology (Finland), Tech. Rep., Sep. 2004.

[33] J. Lu, K.B. Letaief, J.C-I Chuang, and M.L. Liou, "M-PSK and M-QAM BER computation using signal-space concepts," IEEE Transactions on Communications, vol. 47, no. 2, pp. 181-1849, Feb. 1999.

[34] J.G. Proakis and M. Salehi, Digital Communications. New York, McGraw-Hill Higher Education, 2008.

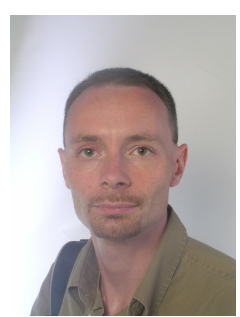

Massimiliano Comisso (M'09) received the Laurea degree in Electronic Engineering and the Ph.D. degree in Information Engineering from the University of Trieste, Italy. He was with Alcatel, where he worked on DWDM communication systems, and collaborated with Danieli Automation on electromagnetic NDE techniques. Currently, Massimiliano Comisso is an Assistant Professor at the Department of Engineering and Architecture (DIA) of the University of Trieste. He is author/co-author of more than 80 international scientific papers, and serves as referee/TPC member for several IEEE journals and conferences. He has been Best Student Paper Award (BPA) Finalist at GLOBECOM 2006 and received the BPA at CAMAD 2009. His research interests involve distributed wireless networks, millimeter-wave communications, antenna array synthesis, and small antennas.

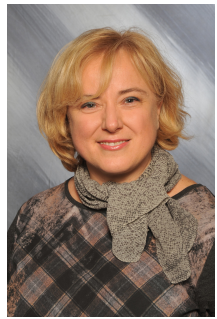

Francesca Vatta (M'98) received the M.Sc. Degree in Electronic Engineering and the Ph.D. Degree in Telecommunications from the University of Trieste, Trieste, Italy, in 1992 and 1998, respectively. She joined the Department of Engineering and Architecture (DIA) of the University of Trieste in 1999, where she is Assistant Professor of Information Theory and Error-Control Coding. Starting in 2002, she spent several months as visiting scholar at the University of Notre Dame, Notre Dame, IN, U.S.A., cooperating with the Coding Theory Research Group under the guidance of Prof. D. J. Costello, Jr. Starting in 2005, she spent several months as visiting scholar at the University of Ulm, Germany, cooperating with the Telecommunications and Applied Information Theory Research Group under the guidance of Prof. M. Bossert. She is an author of more than 100 papers published on international journals and conference proceedings. Her current research interests are in the area of channel coding concerning, in particular, the analysis and design of capacity achieving coding schemes.

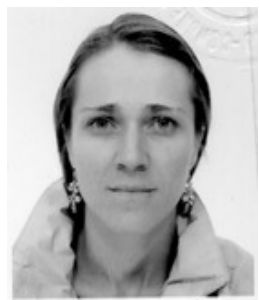

Giulia Buttazzoni received the Laurea degree (summa cum laude) in Telecommunication Engineering and the Ph.D. degree in Information Engineering from the University of Trieste (Italy), in 2008 and 2013, respectively. She is currently a researcher at the Department of Engineering and Architecture of the University of Trieste. Her research interests involve antenna array synthesis and numerical methods for electromagnetic fields.

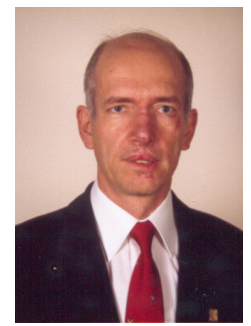

Fulvio Babich (SM'02) received the doctoral degree, (Laurea), cum laude, in Electrical Engineering, at the University of Trieste, on July 1984. After graduation he was with Telettra at the Research and Development Laboratories, where he was engaged in optical fiber communications. Then he joined Zeltron, where he was a communication system engineer, responsible of the activities within the ESPRIT program. In 1992 he joined the Department of Electrical and Electronic Engineering (DEEI) of the University of Trieste, where he is Professor of Digital Communications and Wireless Networks. Currently, Fulvio Babich is vice-director of the Department of Engineering and Architecture (DIA) and coordinator of the Ph.D. program for Industrial and Information Engineering of the University of Trieste. He is also member of the board of the National Telecommunications and Information Theory Group (GTTI), and was member of the Directive Board of the National Inter-University Consortium for Telecommunications (CNIT). His current research interests are in the field of wireless networks and millimeter-wave communications, where he is involved in channel modeling, multiple access techniques, channel encoding, error control techniques, and cross-layer design. 